\title{
Chromatic Number of Graphs with Special Distance Sets-V
}

\author{
Venkataraman Yegnanarayanan', Angamuthu Parthiban ${ }^{2}$ \\ Department of Mathematics, Velammal Engineering College, Chennai, India \\ Email: prof.yegna@gmail.com, mathparthi@gmail.com
}

Received August 22, 2012; revised September 22, 2012; accepted September 30, 2012

\begin{abstract}
An integer distance graph is a graph $G(Z, D)$ with the set of integers as vertex set and an edge joining two vertices $u$ and $v$ if and only if $|u-v| \in D$ where $D$ is a subset of the positive integers. It is known that $\chi(G(Z, P))=4$ where $P$ is a set of Prime numbers. So we can allocate the subsets $D$ of $P$ to four classes, accordingly as $\chi(G(Z, D))$ is 1 or 2 or 3 or 4 . In this paper we have considered the open problem of characterizing class three and class four sets when the distance set $D$ is not only a subset of primes $P$ but also a special class of primes like Additive primes, Deletable primes, Wedderburn-Etherington Number primes, Euclid-Mullin sequence primes, Motzkin primes, Catalan primes, Schröder primes, Non-generous primes, Pell primes, Primeval primes, Primes of Binary Quadratic Form, Smarandache-Wellin primes, and Highly Cototient number primes. We also have indicated the membership of a number of special classes of prime numbers in class 2 category.
\end{abstract}

Keywords: Primes; Chromatic Number; Distance Graphs

\section{Introduction}

The graphs considered in this paper are simple and undirected. A $k$-coloring of a graph $G$ is an assignment of $k$ different colors to the vertices of $G$ such that adjacent vertices receive different colors. The minimum cardinality $k$ for which $G$ has a $k$-coloring is called the chromatic number of $G$ and is denoted by $\chi(G)$. Let $G$ and $H$ be graphs with $H \subseteq G$, then it is easy to see that $\chi(H) \leq \chi(G)$ and so $\chi$ is a monotone function.

We begin with the plane coloring problem. What is the least number of colors needed to color all the points of the Euclidean plane such that no two points of distance one have the same color? The corresponding problem for the real line is easy. $\chi(G(R, D=\{1\}))=2$. To see this, partition the vertex set of $G$ into two non empty disjoint sets such that their union is $R$.

That is, $V(R)=V_{1} \cup V_{2}$, where $V_{1}=\bigcup_{n=-\infty}^{\infty}[2 n, 2 n+1)$ and $V_{2}=\bigcup_{n=-\infty}^{\infty}[2 n+1,2 n+2)$. Now color all the vertices

\begin{tabular}{llllllll}
\hline-3 & -2 & -1 & 0 & 1 & 2 & 3 & 4
\end{tabular}

Figure 1. A sample Distance graph

$G(A=\{-3,-2,-1,0,1,2,3,4\} \subset Z, D)$ with $D=\{1\}$. of $V_{1}$ with color 1 and the vertices of $V_{2}$ with color 2 . As $V_{i}, i=1,2$ are independent and $G(R, D=\{1\})$ is bipartite the result follows. Clearly $G\left(R^{2},\{1\}\right)$ is an infinite graph. The problem of finding the chromatic number of $G\left(R^{2},\{1\}\right)$ is enormously difficult. Paul Erdos has mentioned this problem as one of his favorite problems. Although he could not solve this problem he has made a significant progress towards the solution of this problem with a vital result given as follows: First let us state the famous Rado's Lemma [1].

Lemma 1.1 Let $M$ and $M_{1}$ be arbitrary sets. Assume that to any $v \in M_{1}$ there corresponds a finite subset $A_{v}$ of $M$. Assume that to any finite subset $N \subset M_{1}$, a choice function $x_{N}(v)$ is given, which attaches an element of $A_{v}$ to each element $v$ of $N$ : $x_{N}(v) \in A_{v}$. Then there exists a choice function $x(v)$ defined for all $v \in M_{1}\left(x(v) \in A_{v}\right.$ if $\left.v \in M_{1}\right)$ with the following property. If $K$ is any finite subset of $M_{1}$, then there exists a finite subset $N\left(K \subset N \subset M_{1}\right)$, such that, as far as $K$ is concerned, the function $x(v)$ coincides with $x_{N}(v): x(v)=x_{N}(v)(v \in K)$.

Theorem 1.1 ([2]) Let $k$ be a positive integer, and let the graph $G$ have the property that any finite subgraph is $k$-colorable. Then $G$ is $k$-colorable itself.

Theorem 1.1 allows us to look for the maximum number of colors needed for the finite subsets. It is obvious that $G\left(R^{2},\{1\}\right)$ is not a trivial graph. Therefore 
$\chi\left(G\left(R^{2},\{1\}\right)\right) \neq 1$. The presence of at least one edge, viz., between $(0,0)$ and $(1,1)$ in $G\left(R^{2},\{1\}\right)$ conveys the information that $\chi\left(G\left(R^{2},\{1\}\right)\right) \geq 2$. Similarly, the presence of a clique of size 3 , viz., $K_{3}$ with vertices at $(0,0),\left(\frac{1}{2}, \frac{\sqrt{3}}{2}\right),(1,1)$ shows that

$$
\chi\left(G\left(R^{2},\{1\}\right)\right) \geq 3 .
$$

Moreover, it is a fact that four points in the Euclidean two dimensional plane cannot have pairwise odd integer distances. Therefore, a clique of size 4, viz., $K_{4}$ cannot be an induced subgraph of $G\left(R^{2},\{1\}\right)$. But it will be quite interesting to find the coordinates of a unit distance finite subgraph of $G\left(R^{2},\{1\}\right)$ such that $\chi\left(G_{1}\right)=4$. The Moser Spindle graph in Figure 1 with a chromatic 4-coloring is a good example to deduce that

$$
\chi\left(G\left(R^{2},\{1\}\right)\right) \geq 4 .
$$

It is interesting to note that so far no unit distance graph that requires exactly five colors are known. Falconer [3] showed that if the color classes form meaurable sets of $R^{2}$, then at least five colors are needed. Since the construction of non measurable sets requires the axiom of choice, we might have the answer turn out to depend on whether or not we accept the axiom of choice. We can tile the plane with hexagons to obtain a proper 7 -coloring of the graph. The result is originally due to Hadwiger and Debrunner [4]. So $4 \leq \chi\left(G\left(R^{2},\{1\}\right)\right) \leq 7$.

See Figures 2 and 3 for the lower and upper bounds respectively. Despite the age of this problem, very little progress has been made since the initial bounds on $\chi$ were discovered shortly after the problem's creation. This fact is a testament to the difficulty of the problem and in the absence of progress on the main problem, a number of restricted versions and related questions have been studied. We study here the open problem of characterizing the class 4 sets mentioned in the problem for the prime distance graph whose vertex set is $Z$ and the distance set $D$ is a subset of not only primes $P$ but are also they are a special set of primes. The authors have already made some progress in [5-11].

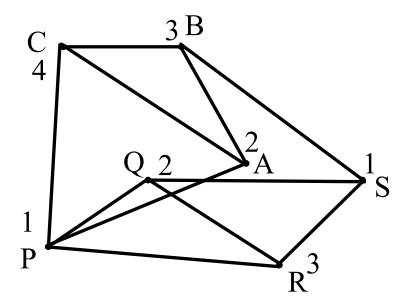

Figure 2. The moser spindle.

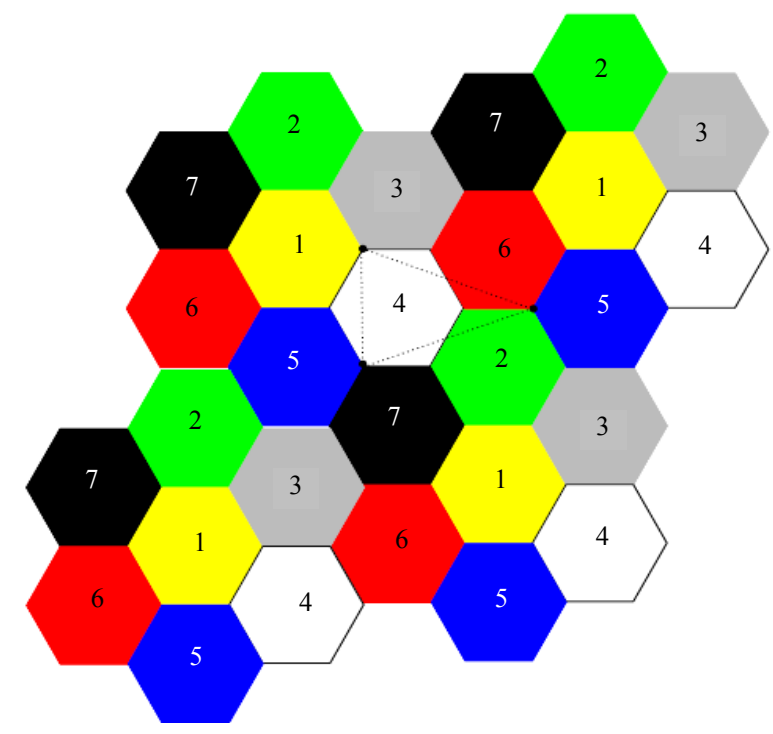

Figure 3. The hexagonal 7 coloring.

\section{Prime Distance Graph}

A prime distance graph is a graph $G(Z, P)$ with the set of integers as vertex set and with an edge joining two vertices $u$ and $v$ if and only if $|u-v| \in P$ where $P$ is the set of all prime numbers.

By a chromatic subgraph of a graph $G$ we mean a minimal subgraph of $G$ with the same chromatic number as $G$. What class of graphs will include a chromatic subgraph of $G(Z, D)$ for $D \subseteq Z$ ?

A graph $G$ is color critical if its only chromatic subgraph is $G$ itself. For any positive integer $m, n$, let $G(m, n)$ be the graph comprising $(m+1)$ distinct vertices $u_{0}, u_{1}, \cdots, u_{m}$ and $m$ distinct subgraphs $H_{1}, \cdots, H_{m}$ each of which is a copy of $K_{n}$, (Where $K_{n}$ is the complete graph on $\mathrm{n}$ vertices) such that $u_{0}$ is adjacent to $u_{m}$ and each vertex of $H_{i}$ is adjacent to $u_{i-1}$ and $u_{i}$, for $1 \leq i \leq m$.

$$
G(m, 1) \cong C_{2 m+1}, G(1, n) \cong K_{n+2},
$$

where $C_{2 m+1}$ is the cycle on $2 m+1$ vertices.

\section{Certain Known Results}

Lemma 2.1 ([12]) For any positive integers $m, n$ the graph $G(m, n)$ is color critical with

$\chi(G(m, n))=n+2$.

Theorem 2.1 ([13]) $\chi(G(Z,\{2,3,5\}))=4$ and hence $\chi(G(Z, P))=4$.

Proof. Let each integer $x$ be assigned a color class $i$ precisely when $x \equiv i(\bmod 4)$, for $0 \leq i \leq 4$. Since integers assigned to the same color differ by a multiple of 4 , they are not adjacent in $G(Z, P)$, so

$$
\chi(G(Z, P)) \leq 4 \text {. }
$$


Since $G(Z,\{2,3,5\}) \subseteq G(Z, P)$ and $\chi$ is monotone, we have

$$
\chi(G(Z,\{2,3,5\})) \leq \chi(G(Z, P)) .
$$

But as $G(Z,\{2,3,5\}) \supseteq G(2,2)$ we have

$$
\chi(G(Z,\{2,3,5\})) \geq 4
$$

where $G(2,2)$ is shown in the Figure 4 determined by the vertices $u_{0}=2, u_{1}=3, u_{2}=4$ and the subgraphs $H_{1}, H_{2}$ with vertex sets $\{0,5\}$ and $\{1,6\}$ respectively. The proof now follows from the Lemma 2.1.

In view of Theorem 2.1, we can allocate the subsets $D$ of $P$ to four classes, according as $G(Z, D)$ has chromatic number $1,2,3$ or 4 . Obviously empty set is the only member of class 1 and every singleton subset is in class 2 .

Lemma $2.2 \chi(G(Z, D))=2$ if $D$ consists only of odd integers.

Proof. Partition $V(Z)$ into two sets with

$$
V(Z)=V_{1}(Z) \cup V_{2}(Z)
$$

such that an integer $x \in V_{i}(Z)$ if and only if $x \equiv i(\bmod 2)$. Now as the elements of $V_{1}(Z)$ are even and the elements of $V_{2}(Z)$ are odd we find that $V_{j}$ is an independent set for $j=1,2$. Therefore $G$ is bipartite and hence $\chi(G(Z, D))=2$. See Figure 5, where $=$ indicates the presence of an edge between any two vertices.

Preposition 1 ([14]) If $D$ is finite and the greatest common divisor of the elements of $D$ is 1 then

(a) $\chi(D)=2$ if all $d_{i} \in D$ are odd,

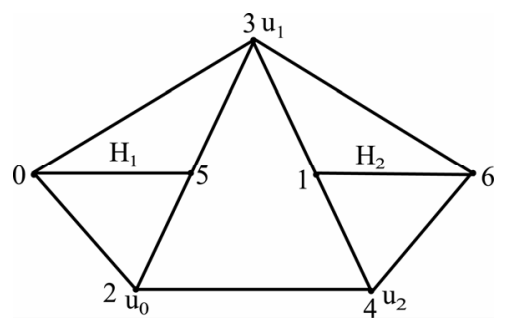

Figure 4. In the figure $H_{1}$ and $H_{2}$ are isomorphic to $K_{2}$.

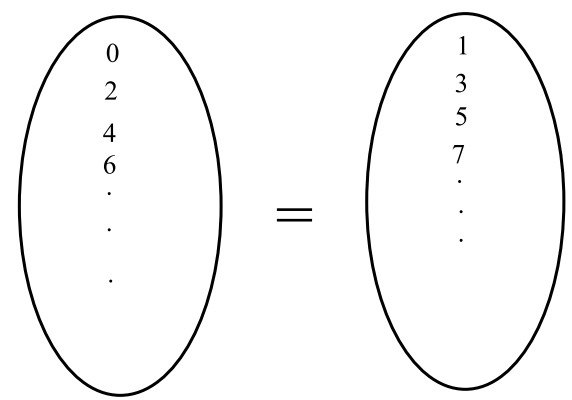

Figure 5. = indicates the presence of an edge between any two vertices of $V_{1}(Z)$ and $V_{2}(Z)$. (b) $\chi(D) \leq 3$ if no distance element is divisible by 3 ,

(c) $\chi(D)=3$ if no distance element is divisible by 3 and at least one distance element is even.

Lemma 2.3 If $D$ contains no multiple of a fixed positive integer $k$, then $\chi(Z, D) \leq k$.

Proof. Color each vertex $x$ in $G(Z, D)$ by $x \bmod$ $k$. Since $x$ and $y$ have the same color if and only if $|x-y|$ is a multiple of $k$, but $D$ contains no multiple of $k$, this is a proper $k$-coloring.

For simplicity, we assume that $\operatorname{gcd}(D)=1$ in the rest of this section. If $|D|=1$, then it is not hard to see that $\chi(Z, D)=2$. For the case of $|D|=2, D=\{a, b\}$, where $a$ and $b$ are relatively prime, and so either they are both odd or they are of opposite parity.

Theorem 2.2 If $a$ and $b$ are relatively prime positive odd integers, then $\chi(Z,\{a, b\})=2$.

Proof. The theorem follows immediately from Lemma 2.3 and the fact that $\chi(Z,\{a, b\}) \geq 2$ for any nonempty $D$.

Theorem 2.3 ([13]) Let $D \subseteq P$, with $|D| \geq 2$. Then $D$ may be classified as follows:

(a) $D$ is in class 2 if $2 \notin D$; otherwise $D$ is in class 3 or 4 .

(b) If $2 \in D$ and $3 \notin D$, then $D$ is in class 3 .

(c) If $\{2,3\} \subseteq D \subseteq\{p \in P: p \equiv \pm 2(\bmod 5)\}$, then $D$ is in class 3 .

(d) If $\{2,3\} \subseteq D \subseteq\{p \in P: p \equiv \pm 2, \pm 3,7(\bmod 14)\}$, then $D$ is in class 3 .

(e) If $\{2,3,5\} \subseteq D$ or $\{2,3,11,13\} \subseteq D$, then $D$ is in class 4 .

Theorem 2.4 ([13]) For any prime $p>5,\{2,3, p\}$ is in class 3.

Lemma 2.4 ([15]) There exists only a finite number of sets $D=\{2,3, p, q\}, \quad p, q \in P, \quad p \geq 7$ and $q>p+2$, with $\chi(D)=4$.

Theorem 2.5 ([15]) Let $D=\{2,3, p, q\}$ be a set of primes with $p \geq 7$ and $q>p+2$. Then $\chi(D)=4$ holds if and only if

$$
\begin{gathered}
(p, q) \in\{(11,19),(11,23),(11,37),(11,41), \\
(17,29),(23,31),(23,41),(29,37)\} .
\end{gathered}
$$

The proofs of Lemma 2.4 and Theorem 2.5 are available in [15]. Moreover, the authors M. Voigt and $\mathrm{H}$. Walther have shown that there are exactly eight pairs of primes $p, q \quad(p \geq 7, q>p+2)$ with $\chi(2,3, p, q)=4$.

Theorem 2.6 ([16]) Let $D=\{a, b, c\}$ with $a<b<c$ and $\operatorname{gcd}(a, b, c)=1$. Then

$$
\chi(D)=\left\{\begin{array}{l}
2, \text { if } a, b, c \text { are odd; } \\
4, \quad \text { if } D=\{1,2,3 m\}, \text { or } c=a+b \\
\quad \text { and } b-a \neq(\bmod 3) ; \\
3, \text { otherwise. }
\end{array}\right.
$$




\section{Some Special Set of Prime Numbers}

Consider the following interesting set of prime numbers namely Additive Primes, Deletable Primes, WedderburnEtherington Number Primes, Euclid-Mullin Sequence Primes, Motzkin Primes, Schröder Primes, Catalan Primes, Non-generous Primes, Pell Primes, Primes of Binary Quadratic Form, Primeval Primes, Smarandache-Wellin Primes, Highly Cototient Number Primes, Annihilating Primes, Euclid primes, Fortunate Primes, SchröderHipparchus Number primes, Gaussian Primes, Mersenne Primes, Odd Primes, Primorial Primes, Proth Primes, Regular Primes, Self Primes, Solinas Primes, Super Primes, Delannoy Number Primes, Unique Primes, and Wagstaff Primes. For the definitions of these set of primes refer [17]. However, the existence of an interesting mathematical process that logically leads to the precise definition of an Annihilating prime we mention the same here for the sake of completeness and for the ease of the readers. Each of these set of primes have certain unique properties that make them special and are found to be useful from both theoretical and practical point of view.

\section{Annihilating Primes}

Definition 3.1 ([18]) For $n \in N=\{0,1,2, \cdots\}$ let $\operatorname{seq}^{1-1}(n)$ denote the number of one-to-one sequencesthese are sequences without repetitions-we can build with $n$ distinct objects.

\langle\rangle (= the empty sequence), $\left\langle a_{1}\right\rangle,\left\langle a_{2}\right\rangle,\left\langle a_{1}, a_{2}\right\rangle$, $\left\langle a_{2}, a_{1}\right\rangle$.

Hence, $\operatorname{seq}^{1-1}(2)$. Of course, it is easy to find a general expression for $\operatorname{seq}^{1-1}(n)$. Since there are $\left(\begin{array}{l}k \\ n\end{array}\right)$ possible ways to choose $k$ objects from a set of $n$ (distinct) objects, and since $k$ (distinct) objects give rise to $k$ ! permutations, we get the following Lemma.

Lemma $3.1 \operatorname{seq}^{1-1}(n)=\sum_{k=0}^{n}\left(\begin{array}{l}k \\ n\end{array}\right) k !=\sum_{j=0}^{n} \frac{n !}{j !}$. Also the next representation for $\operatorname{seq}^{1-1}(n)$ is elementary.

Lemma 3.2 For all positive $n \in N$ we have $\operatorname{seq}^{1-1}(n)=\lfloor$ en! $\rfloor$.

Remark: For $n=0$ the formula does not hold, since $\operatorname{seq}^{1-1}(0)=1<2=\lfloor e 0 !\rfloor$.

Proof. According to Lemma 3.1 we have

$$
\begin{aligned}
\text { en } & =\operatorname{seq}^{1-1}(n)+\sum_{j=n+1}^{\infty} \frac{n !}{j !} \\
& =\operatorname{seq}^{1-1}(n)+\underbrace{\frac{1}{n+1}\left(1+\frac{1}{n+2}+\frac{1}{(n+2)(n+3)}+\cdots\right)}_{\leq \frac{1}{n+1}(e-1)<1 \text { for } n \geq 1}
\end{aligned}
$$

The following recursive relation for $\operatorname{seq}^{1-1}(n)$ is an immediate consequence of the second formula in Lemma 3.1 .

Lemma 3.3 For all positive $n \in N$ we have $\operatorname{seq}^{1-1}(n)=n \operatorname{seq}^{1-1}(n-1)+1$. Using this formula, we finally get the following integral representation of $\operatorname{seq}^{1-1}(n)$.

Lemma 3.4 For all $n \in N$ we have

$$
\operatorname{seq}^{1-1}(n)=e \int_{1}^{\infty} t^{n} e^{-t} \mathrm{~d} t
$$

Notation: Throughout this text we adopt the standard notation $a \mid b$ to express that $a$ divides $b$ for $a, b \in N$. Moreover, if $b \geq 1$ then

$$
\operatorname{Mod}(a, b):=a-b\left\lfloor\frac{a}{b}\right\rfloor
$$

denotes the reminder of the division of $a$ by $b$; and $(a, b)$ denotes the greatest common divisor of $a$ and $b$.

We start our investigation on divisibility properties of $n^{*}$ with a simple fact which has first been proved in [19].

Lemma 3.5 For natural numbers $n, k \in N$, the following implication holds: If $2^{k} \mid n^{*}$, then $2^{k} \mid\left(n+2^{k}\right)^{*}$ and $2^{k} \times(n+t)^{*}$ for any $t$ with $0<t<2^{k}$.

Definition 3.2 If $\{f(n)\}_{n \in N}$ is a sequence of natural numbers, we define its shadow to be the sequence $\{d(h)\}_{h \in N}$ given by $d(h):=|D(h)|$, where

$$
D(h):=\{n \in N:(n<h) \wedge(h \mid f(n))\}
$$

are the shadow sets of the sequence $f$.

The shadow $d(h)$ counts the sequence entries $f(0), f(1), \cdots, f(h-1)$ which are divisible by $h$. So, the shadow measures (to a certain extent) how "divisible" the entries of the sequence $f(n)$ are: For example, if only prime numbers occur in the sequence, then its shadow will reflect this fact by being small. If the entries of $f(n)$ have many divisors, the shadow will typically be large.

If $f(n)$ is an arithmetic sequence of first order, then its shadow is periodic, and for the shadow of Euler's $\varphi$-function we have $d(h)=1$ for all $h \geq 1$.

Example: If for a function $f$ there exists an $n_{0} \in N$ such that for all $h \geq n_{0}$ we have $d(h)=0$, then for all $h \geq n_{0}$ we have $f(h) \leq h$. Vice versa, if $f(h) \leq h$ for all $h \in N$, then $d(h)$ equals the number of zeros in $(f(0), f(1), \cdots, f(h-1))$. Hence, it is easy to construct different functions which have the same shadow:

$$
\begin{aligned}
& n \quad 01234567 \cdots \\
& f_{1}(n) \quad 01234567 \cdots \\
& f_{2}(n) \quad 011123456 \cdots
\end{aligned}
$$




\section{$f_{3}(n) 01112345 \cdots$}

shadow $011111111 \cdots$

Definition 3.3 A sequence $\{f(n)\}_{n \in N}$ is said to have the reduction property, if for all $n, q \in N, q \geq 1$, we have, $\operatorname{Mod}(f(n), q)=\operatorname{Mod}(f(\operatorname{Mod}(n, q)), q)$.

Lemma 3.6 The sequence $\left\{\operatorname{seq}^{1-1}(n)\right\}_{n \in N}$ has the reduction property.

Lemma 3.7 The shadow $d$ of a sequence $f(n)$ which has the reduction property is multiplicative, i.e. if $(a, b)=1$, then $d(a b)=d(a) d(b)$.

As an immediate consequence we get the following:

Corollary 1 If $d$ is the shadow of $\operatorname{seq}^{1-1}$ and if

$$
n=\prod_{i=1}^{k} p_{i}^{k_{i}}
$$

is the prime decomposition of $n$, then

$$
d(n)=\prod_{i=1}^{k} d\left(p_{i}^{k_{i}}\right) .
$$

Therefore, the shadow $d$ of $s e q^{1-1}$ is fully determined by its values on the powers of prime numbers. But what can we say about $d\left(p^{k}\right)$ for $p$ prime?

By the reduction property, all elements $m \in D\left(p^{k+1}\right)$ must be of the form $m=n+l p^{k}$ for some $n \in D\left(p^{k}\right)$ and some $l \in\{0,1, \cdots, p-1\}$. Hence, we get inductively that if $d(p)=0$, then $d\left(p^{k}\right)=0$ for all positive $k \in N$.

Definition 3.4 A prime number $p$ with $d(p)=0$ is called annihilating.

Preposition 2 If $n \in N$ is divisible by an annihilating prime, then $d(n)=0$.

Annihilating primes are primes such that $d(p)=0$, where $d(p)$ is the shadow of a sequence of natural numbers. First few such primes are: 3, 7, 11, 17, 47, 53, $61, \cdots$

\section{Chromatic Number of Certain Prime Distance Graphs}

Here we compute the chromatic number of the distance graph: $G(Z, D)$, when $D$ is a set/subset of any of the above listed primes.

Theorem $4.1 \chi(G(Z, D))=4$, when $D$ is a finite/ infinite set/subset as the case may be of WedderburnEtherington Number Primes.

Proof. Let A denote the set of Wedderburn-Etherington Number Primes. Note that in $A$, the set of primes $\{2,3,11,23\}$ appears as a proper subset. By Lemma 2.4 and Theorem 2.5, the set of primes $\{2,3,11,23\}$ is one of the eight sets of chromatic number 4 class. So

$$
\begin{aligned}
4 & =\chi(G(Z,\{2,3,11,23\})) \\
& \leq \chi(G(Z, D)) \leq \chi(G(Z, P))=4
\end{aligned}
$$

as $\{2,3,11,23\} \subseteq D \subseteq P$.

Theorem $4.2 \chi(G(Z, D))=4$, when $D$ is a finite/ infinite set/subset as the case may be of Additive Primes, Deletable Primes, and Euclid-Mullin sequence primes.

Proof. Let A be the set of Additive primes/Deletable primes/ Euclid-Mullin sequence primes. As the set of primes $\{2,3,5\}$ is a proper subset of $A$, by Theorem 1.1 and Theorem 2.1

$$
\begin{aligned}
4 & =\chi(G(Z,\{2,3,5\})) \\
& \leq \chi(G(Z, D)) \leq \chi(G(Z, P))=4
\end{aligned}
$$

as $\{2,3,5\} \subseteq D \subseteq P$.

Theorem $4.3 \chi(G(Z, D))=3$, when $D$ is a finite/ infinite set/subset as the case may be of 1) Motzkin primes, 2) Catalan primes, 3) Schröder primes, 4) Nongenerous primes, 5) Pell primes, 6) Primeval primes, 7) Primes of binary quadratic form, 8) Smarandache-Wellin primes, and 9) Highly Cototient number primes.

Proof. Let $A$ denote the set of any one of these special class of prime numbers listed in the theorem. All these set of primes have a unique property that an even prime integer 2 is in $A$ but the odd prime integer 3 is not appearing in $A$. So by Theorem 2.3(b) and Preposition 1(c), $\chi(G(Z, D))=3$.

Theorem 4.4 $\chi(G(Z, D))=2$, when $D$ is a finite/ infinite set/subset as the case may be of 1) Euclid primes, 2) Fortunate primes, 3) Schröder-Hipparchus Number primes, 4) Gaussian primes, 5) Mersenne primes, 6) Odd primes, 7) Primorial primes, 8) Proth primes, 9) Regular primes, 10) Self primes, 11) Solinas primes, 12) Super primes, 13) Delannoy Number primes, 14) Unique primes, 15) Wagstaff primes, and 16) Annihilating primes.

Proof. Let A denote the set of any one of these special class of prime numbers listed in the theorem. All these special class of primes have a unique property that $A$ does not contain the even prime integer 2 but $A$ has an odd prime integer 3. So by Theorems 2.2 and 2.3(a), $\chi(G(Z, D))=2$.

\section{Conclusion}

A complete characterization of class three sets and class four sets are available in the literature when the cardinality of the distance subset $D$ of $P$ are either 3 or 4. However when the cardinality of $D$ is more than 4 only a little is known. So in this aspect Theorem 4.1, Theorem 4.2 and Theorem 4.3 makes some progress towards the complete characterization of class three and class four sets. Moreover, Theorem 4.4 brings out certain 
special classes of prime numbers with membership in class 2 category.

\section{Acknowledgements}

This research is carried out with the financial grant and support of National Board for Higher Mathematics, Government of India under the grant sanction no. 2/ 48(10)/2005/R\&D-II/11192/dated 26,Nov,2010.

\section{REFERENCES}

[1] R. Rado, "Axiomatic Treatment of Rank in Infinite Sets," Canadian Journal of Mathematics, Vol. 1, No. 1949, 1949, pp. 337-343. doi:10.4153/CJM-1949-031-1

[2] N. G. de Bruijn and P. Erdos, "A Color Problem for Infinite Graphs and a Problem in the Theory of Relations," Proceedings, Series A, Vol. 54, No. 5; Indagationes Mathematicae, Vol. 13, No. 5, 1951, pp. 371-373.

[3] K. J. Falconer, "The Realization of Distances in Measurable Subsets Covering $R^{n}$," Journal of Combinatorial Theory, Series A, Vol. 31, No. 2, 1981, pp. 184-189. doi:10.1016/0097-3165(81)90014-5

[4] H. Hadwiger and H. Debrunner, "Combinatorial Geometry in the Plane," Holt, Rinehart and Winston, New York, 1964.

[5] V. Yegnanarayanan, "On a Question Concerning Prime Distance Graphs," Discrete Mathematics, Vol. 245, No. 1-3, February 2002, pp. 293-298. doi:10.1016/S0012-365X(01)00221-7

[6] V. Yegnanarayanan and A. Parthiban, "Chromatic Number of Certain Graphs," Proceedings of International Conference on Mathematics in Engineering and Business Management, Vol. 1, Chennai, 9-11 March 2012, pp. 115118.

[7] V. Yegnanarayanan, "Chromatic Number of Graphs with Special Distance Sets, I," Algebra and Discrete Mathematics, Accepted for Publication in January 2013, to appear.

[8] V. Yegnanarayanan and A. Parthiban, "The Chromatic Number of Graphs with Special Distance Sets-III," Jour- nal of Mathematical and Computational Science, Vol. 2, No. 5, 2012, pp. 1257-1268.

[9] V. Yegnanarayanan, "The Chromatic Number of Generalized Fibonacci Prime Distance Graph," Journal of Mathematical and Computational Science, Vol. 2, No. 5, 2012, pp. 1451-1463.

[10] V. Yegnanarayanan and A. Parthiban, "The Chromatic Number of Graphs With Special Distance Sets-II," Proceedings of International Conference on Mathematical Modelling and Applied Soft Computing, CIT, Vol. 1, Coimbatore, 11-13 July 2012, pp. 305-313.

[11] V. Yegnanarayanan and A. Parthiban, "The Chromatic Number of Graphs With Special Distance Sets-IV," Proceeding of International Conference on Applied Mathematics and Theoretical Computer Science, Kanyakumari, 2013, to appear.

[12] R. B. Eggleton, P. Erdos and D. K. Skilton, "Coloring the Real Line," Journal of Combinatorial Theory, Series B, Vol. 39, No. 1, 1985, pp. 86-100; To Erratum, Vol. 41, 1986, p. 139.

[13] R. B. Eggleton, P. Erdos and D. K. Skilton, "Colouring Prime Distance Graphs," Graphs and Combinatorics, Vol. 6, No. 1, 1990, pp. 17-32. doi:10.1007/BF01787476

[14] A. Kemnitz and H. Kolberg, "Coloring of Integer Distance Graphs," Discrete Mathematics, Vol. 191, No. 1-3, 1998, pp. 113-123. doi:10.1016/S0012-365X(98)00099-5

[15] M. Voigt and H. Walther, "Chromatic Number of Prime Distance Graphs," Discrete Applied Mathematics, Vol. 51, No. 1-2, 1994, pp. 197-209. doi:10.1016/0166-218X(94)90109-0

[16] X. Zhu, "The Circular Chromatic Number of Distance Graphs with Distance Sets of Cardinality 3," Journal of Graph Theory, Vol. 41, No. 3, 2002, pp. 195-207. doi:10.1002/jgt.10062

[17] en.wikipedia.org/wiki/List_of_prime_numbers

[18] L. Halbeisen1 and N. Hungerbuhler, "Number Theoretic Aspects of a Combinatorial Function," 2000. www.math.uzh.ch/user/halbeis/publications/pdf/seq.pdf

[19] L. Halbeisen and S. Shelah, "Consequences of Arithmetic for Set Theory," Journal of Symbolic Logic, Vol. 59, No. 1, 1994, pp. 30-40. doi: $10.2307 / 2275247$ 